\title{
De la presencia impuesta a la presente ausencia: traducción de la teoría cultural rusa en Cuba por Desiderio Navarro (I960-2009)
}

\author{
From Imposed Presence to Present Absence: Desiderio Navarro's \\ Translation of Russian Cultural Theory in Cuba
}

\begin{abstract}
RaÚL ERNESTO COLÓN RODRÍGUEZ
Universidad de Ottawa·rcolo036@uottawa.ca

Candidato al doctorado en traductología y profesor a tiempo parcial en la Universidad de Ottawa. Ha trabajado la traducción editorial y cinematográfica en Canadá. Ha publicado artículos y reseñas en: TTR, META, Glendon Selected Proceedings 2013; Aspectos de la historia de la traducción en Hispanomérica: autores, traducciones y traductores ; Belas Infieis ; Mutatis Mutandis.
\end{abstract}

RECIBIDO: 16 DE FEBRERO DE 2015

ACEPTADO: 5 DE JUNIO DE 2015

Resumen: En este artículo, se pretende primero responder a las cuestiones relativas a la selección de textos y autores por parte del traductor Desiderio Navarro, para la antología del pensamiento ruso que logra publicar en Cuba en 2009. Se pretende también explicar los contextos y las contradicciones inherentes a los procesos sociales, ideológicos y políticos en la ex- URSS y en la Cuba de los últimos 56 años. Este estudio parte de la hipótesis que Navarro ha sido y es un intelectual crítico, en el sentido que ese concepto fue interpretado en Cuba desde los años 1960, es decir, que junto con el reclamo de un lugar activo y cuestionador del poder para este sector social, se integra y defiende una fidelidad sin límites tanto a los postulados de un marxismo no ortodoxo, como a los del paradigma nacionalista que ha caracterizado al pensamiento de
Abstract: This essay attempts first, to answer questions related to the choice of texts and authors, selected by the translator and editor Desiderio Navarro for his anthology of Russian theory published in 2009, in Cuba. Then, to explicit the context and contradictions that were implicit to the social, ideological and political processes in the ex-USSR, and in Cuba. This study's key hypothesis is that Navarro was, and still is, a critical intellectual, in the same sense this concept was interpreted in 1960's Cuba. This means that together with the claim of an active and critical role vis-à-vis the Power, there is also an unconditional fidelity to the ideas of nonorthodox Marxism and to the nationalistic paradigm, both key elements of Cuba's contemporary social thinking.

Key Words: Desiderio Navarro, antology, Russian thoughts, translation, ex-USSR, Cuba. 


\section{Introducción}

El estudio de la selección de textos teóricos, de conjunto con el análisis de algunos elementos principales de la trayectoria de un agente (Bourdieu, 1994), a la vez traductor y teórico, tiene como objetivo contribuir al conocimiento de su obra y al debate en torno a la misma ${ }^{1}$. Esa obra, desde hace ya más de 50 años, tanto en el contexto cubano como en el hispanoamericano y brasileño, ha visto aumentar su influencia. Desiderio Navarro ha venido realizando un considerable trabajo de traducción y ha publicado una obra teórica propia inspirada de los numerosos textos teóricos que ha traducido, en particular de autores rusos, ellos mismos polémicos y hasta contestatarios de un discurso dominante, es decir, del marxismo estalinista transformado en doxa supranacional ${ }^{2}$. Este artículo muestra que en América latina en los siglos XX y XXI, como ya había sido el caso en el siglo XIX, la decisión de traducir ciertos textos de carácter social, teórico y político -y no solamente la traducción como tal, en generalcontinua presentando la tendencia a la apropiación que ha sido constatada en historia de la traducción en esta región del mundo (Bastin \& Echeverri, 2004). Apropiación que consiste, según estos autores en "[...] suscitar un desplazamiento de autoría o de propiedad, es decir, de carácter propio; un desplazamiento en el que lo "propio" cambia de manos" (2004: 70). Veremos entonces en el caso que nos ocupa, que desde que se selecciona un texto para traducir y publicar, o republicar, en un momento dado, estamos en presencia de un acto de apropiación. Este estudio muestra también que la trayectoria de este traductor y teórico cubano representa, de un lado, una lucha permanente contra doxas tanto nacionales como internacionales ${ }^{3}$ y de otro un esfuerzo sostenido de difusión de nuevas teorías e ideas, así como de estímulo a la creación teórica y artística. Navarro ha también jugado un rol clave como

\footnotetext{
${ }^{1}$ Para mayor información sobre este tema ver la tesis de maestría en traductología del autor: La traduction de la pensée culturelle russe par Desiderio Navarro à Cuba, 1960-2009 (Colón Rodríguez, 2011).

${ }^{2}$ El concepto de doxa supranacional se aplica aquí al marxismo de factura stalinista, promovido e impuesto entre 1924 y formalmente- 1956, no solamente en la URSS sino también en los países del Bloque del Este y en otros países aliados noeuropeos, así como en la mayor parte de los partidos comunistas de todo el mundo. Esta variante ultra-ortodoxa del marxismo sobrevivirá la parcial crítica jruschoviana mucho más allá de 1956, no siendo verdaderamente cuestionada sino con la llegada de la Perestroika gorbachoviana. Por su parte, el concepto de doxa hace referencia aquí a Aristóteles en el sentido de: « opinions partagées par tous les hommes, ou par presque tous, ou par ceux qui présentent l'opinion éclairée, et pour ces derniers par tous, ou par presque tous, ou par les plus connus et les mieux admis comme autorités » (opiniones compartidas por todos los hombres, o por casi todos, o por aquellos que ofrecen una visión clara, y para estos últimos por todos, o por casi todos, o por los más conocidos y mejor aceptados como autoridades). Cita de Aristóteles (Aristote, Topiques: I, 1) en el artículo « Doxa » (Charaudeau et Maingueneau 2002: 197). Todas las traducciones del francés u otras lenguas en este artículo son del autor, salvo se diga lo contrario.

${ }^{3}$ Es oportuno recordar aquí que el marxismo stalinista no fue sino uno de los discursos dominantes del siglo XX, junto con el fascista, el neoliberal, el neoconservador, el neoreligioso y también el neomarxismo, que sigue haciéndole la competencia al primero hasta nuestros días. Ver también: Judt \& Snyder, 2012.
} 
promotor y organizador de la protesta de los intelectuales del 2007 en Cuba, y como intermediario ante el poder y sus representantes. El rol de mediador cultural, social y político que este traductor asume es permanente.

La hipótesis avanzada es que la selección, traducción y apropiación de textos y teorías rusas por Navarro, en una época en que tanto unos como otras eran más bien marginales en su propio contexto, y poco conocidos a nivel internacional, tenía como objetivo primordial contrarrestar el dogmatismo soviético y marxista local en los medios universitarios cubanos. Como consecuencia también estimular y favorecer un cambio de mentalidad para, en última instancia, contribuir a un cambio social a través de la reemergencia del intelectual crítico, en el sentido que ese concepto fue interpretado en Cuba desde los años 1960 por la oposición lealt. Esto quiere decir que, junto con el reclamo de un lugar activo y cuestionador del poder para este sector social, se integra y defiende una fidelidad sin límites tanto a los postulados de un marxismo no ortodoxo, como a los del paradigma nacionalista que ha caracterizado al pensamiento de la Cuba contemporánea. Veremos también que estos propósitos son ampliamente sobrepasados por el impacto real que ha tenido la obra navarriana.

En cuanto a los límites del estudio, es preciso señalar que se trata del análisis de la traducción al español por Navarro de una parte de la teoría literaria y lingüística ruso-soviética del pasado siglo por él realizada. Por razones de espacio nos circunscribimos a 5 de los 33 textos publicados en la antología $E l$ Pensamiento Cultural Ruso en criterios. $1972-2008^{5}$, no obstante los 33 textos de esta antología han sido ampliamente consultados. El mismo ha constituido el corpus de una tesis de maestría en traductología, defendida en la Universidad de Ottawa en 2011 (Colón Rodríguez, 2011).

\section{La selección de los textos. Contexto (cubano) y textos (rusos)}

Los autores seleccionados por Desiderio Navarro para la antología del pensamiento ruso que publicó en Cuba en 2009 son hoy reconocidos internacionalmente, tanto por la calidad analítica de sus trabajos como por los aportes teóricos a sus respectivas disciplinas académicas, pero cuando el traductor

\footnotetext{
${ }^{4}$ Este término reemerge en la actualidad en Cuba, con el declive pronunciado del capital simbólico de la ideología oficialista y la tolerancia relativa de puntos de vista divergentes, de parte de intelectuales que son en diferente medida orgánicos al proceso. Ver aquí.

5 Se respeta aquí y en todo el artículo la ortografía del título de la antología, pues tiene una intencionalidad bien definida. Navarro publica en los dos volúmenes de la antología solamente 33 textos de un total de 115 de autores teóricos rusos que él ha traducido. Esos textos tocan temas tan diversos como la literatura, el cine, el teatro, las artes plásticas, la estética y la culturología y en buena parte están disponibles en el sitio Web de la revista Criterios.
} 
y antologista cubano los tradujo y publicó por primera vez $z^{6}$, no lo eran tanto, de hecho Navarro ha sido pionero al hacerlo hacia el español en la mayor parte de los casos. Mijaíl Bajtín, Yuri Lotman, Eleazar Meletinski, Pavel Medvedev, Boris Groys, entre muchos otros, también fueron científicos que experimentaron en su país natal o de adopción el ostracismo por parte de las autoridades soviéticas, de modo que estos textos eran necesariamente o más bien lógicamente contestatarios de una doxa que no solamente había tratado de eliminar todo pensamiento adverso dentro y fuera del campo del poder, en particular con el arribo al poder en 1924 de Stalin, sino también todo pensamiento creativo, dialectico o dialógico de filiación marxista. En la conciencia social de los pueblos soviéticos y de sus élites se va a inculcar la idea de un "Estado eterno" (Yurchak, 2007: 1), que nada ni nadie podrá ya destruir. A todos los niveles de la sociedad la duda o el cuestionamiento de los postulados de la ideología comunista van a ser combatidos tanto o más que la disidencia política. Ello explica que en la URSS la norma fuese exigir de los universitarios y científicos una fidelidad ideológica casi religiosa a la interpretación, que de las obras de Marx, Lenin y otros autores clásicos del marxismo, emanaban de Stalin. Esto es algo que Yurchak, citando a Erving Goffman llama el rol de "principal del discurso", es decir "alguien que se posiciona fuera del discurso, pudiendo evaluarlo públicamente e inclusive sugerir cómo mejorarlo" (2003: 488). Después de la muerte de Stalin, será el Departamento ideológico del Comité Central del PCUS (partido comunista de la Unión Soviética), quien tomará el batón de relevo, operándose lo que también Yurchak califica de "desplazamiento heteronímico", es decir, un fenómeno en el que "la forma queda intacta y el cambio tiene lugar a nivel del sentido" (2003: 481). Es ese desfasaje semántico el que va a provocar, junto a otros factores materiales, una gradual estagnación y degradación de la sociedad soviética. El disenso ante la exigencia de fidelidad a los postulados ideológicos promovidos desde el poder durante ambas etapas de la historia soviética, sigue siendo combatido por medio de una represión que gana en sofisticación durante los gobiernos de Jruschov, Brezhnev y los restantes primeros secretarios del PCUS ${ }^{7}$, hasta el cambio radical de esa política que va a significar la Perestroika gorbachoviana. Constataremos que algunos de los autores incluidos en la antología son disidentes que se escaparon a Occidente ${ }^{8}$ y es bien conocido que en los países socialistas

\footnotetext{
${ }^{6}$ Navarro comienza a traducir y a publicar estos autores fundamentalmente a partir de la creación de la revista Criterios en 1973.

${ }^{7}$ Después de la muerte de Brezhnev se suceden varios primeros secretarios de "corta duración", debido a la edad avanzada de los miembros del Buró político del PCUS en esa época. La llegada al poder de Mijaíl Gorbachov en 1985 significa el cambio generacional decisivo para romper la inercia observada hasta entonces en la cima del poder soviético. Ver también: Blanc et Lesnik, 2009.

${ }^{8}$ Es el caso de Boris Groys, quien nació en Berlín oriental en 1947, pero que vive “en Rusia hasta los años 1980. Estudia matemáticas y filosofía en la Universidad de Leningrado. Es allí asistente de investigación, enseña luego lingüística en Moscú y se convierte en un crítico del sistema comunista. Se le prohíbe viajar a Occidente. En 1981 se escapa y se instala en la República federal de Alemania [...]”. (Información tomada del sitio Web personal de Groys. Ver: Referencias).
} 
aliados de Moscú esa actitud siempre fue seguida de una censura completa de sus obras. El hecho que esta antología haya sido publicada en Cuba y que comience por el texto de un antiguo disidente (Boris Groys), muestra la importante erosión de los viejos postulados stalinistas en la isla. Muestra también que los regímenes socialistas que sobrevivieron la debacle de 1989-1991, como es el caso del cubano, lo hacen al precio de ciertas concesiones y Navarro en el prefacio de la antología agrega:

La compilación que ponemos en manos del lector cubano no es una antología en el sentido habitual de una recopilación de textos escogidos con un mismo propósito editorial en un único momento en el tiempo. [...] Debo aclarar, de entrada, que esas sucesivas selecciones anteriores nunca pudieron ser realizadas sobre el conjunto del corpus teórico-cultural ruso realmente existente en cada momento dado, sino que fueron pequeños logros alcanzados en una lucha individual con limitaciones informativas, económicas e institucionales, tanto en Cuba como en la URSS y luego en la Rusia postsoviética (2009: v)

Con motivo de contextos de emisión y de recepción ${ }^{9}$ complejos, Navarro enfrenta numerosos obstáculos para poder acceder a los libros, teniendo a veces que copiarlos a mano, para luego traducirlos, pero es evidente que se encuentra en una dinámica en la que posee acceso a los textos. Disfruta además de los numerosos y frecuentes viajes que hacen los intelectuales y funcionarios cubanos de la cultura a la URSS en los años 1970 y 1980, invitados a festivales y congresos internacionales, para establecer contacto con esos autores y esos textos. Navarro comprende muy rápido que en la URSS también hay voces discordantes con el aparente monolítico discurso dominante, voces que discuten ideas de gran interés para el debate cultural y teórico cubano, de modo que él decide canalizar su voluntad de recuperación del debate en Cuba a través de la traducción de textos de crítica literaria y culturológica de países de Europa del Este que ya han recorrido un camino considerable en la construcción de sociedades socialistas, algo que en Cuba sólo se acaba de comenzar y con bastantes tropiezos desde la década de los 1960 .

\footnotetext{
${ }^{9}$ Bajo el concepto de recepción se sobreentiende aquí una "expresión empleada por todo enfoque literario que se interesa en la recepción de obras literarias, ya sea desde una perspectiva psicológica o sociológica”, (van Gorp y col., 2001 : 404), cercana a la visión de la investigación empírica o experimental de la recepción de N. Groeben o de S.J. Schmidt (van Gorp y col., 2001: 406), pero para ser más precisos voy a retomar la cita-resumen que hace Henryk Markiewicz (traducido al español por Navarro), de los estudios contemporáneos de la recepción: “con frecuencia cada vez mayor, y desde distintas posiciones, la obra literaria es definida metafóricamente como «encuentro» (R. Ingarden), «diálogo» (M. Bajtín), «convergencia» (H. R. Jauss), o «interacción» (W. Iser) entre el texto y el lector" (Markiewicz, 2010: 46). Navarro, traductor de Markiewicz y fino conocedor de los textos sobre la recepción, aplica en sus traducciones y trabajos teóricos la divisa de Markiewicz que por demás cita en el prefacio de ese volumen consagrado a Markiewicz: "La tarea capital de la interpretación no es el acercamiento de la obra al lector, sino el acercamiento del lector a la obra", (Markiewicz, 2010: 7). Esa idea, íntimamente asociada en traductología al aporte de Schleiermacher (1999 [1813]), en su célebre texto: « Des différentes méthodes de traduire », es un postulado que combate el nivelamiento hacia un mínimo común denominador, propio al contexto cubano contemporáneo.
} 
Ese trabajo de traducción le permite enfrentar otro trabajo, esta vez teórico y en ese marco va a apropiarse y utilizar categorías y términos que ha traducido de los trabajos de esos autores rusos y del Este $^{10}$.

Por otro lado, la posibilidad de elegir los textos que se quiere traducir no es algo de lo cual todos los traductores pueden vanagloriarse. Construir, además, a lo largo de toda una carrera un vasto corpus de traducciones de carácter teórico es, no cabe duda, un desafío considerable. Navarro, no solamente en el marco cubano, sino también en el internacional, es un raro ejemplo de éxito en ambos campos. En Cuba la mayor parte de los traductores trabajan en instituciones estatales después de 1959 y traducen textos que no constituyen una opción personal. Esos textos poseen con frecuencia, cuando se trata de aquellos relativos a las ciencias sociales, una tendencia ideológica bien definida, en consonancia, por cierto, con uno de los objetivos centrales de la Asociación de traductores del país ${ }^{11}$. Los profesionales de la traducción más mediatizados son los intérpretes del poder, en particular del jefe de gobierno ${ }^{12}$.

Es por esa razón que el caso de Navarro es atípico, pues reúne varios aspectos extremadamente singulares, como son, primero: haber conseguido convertirse en traductor de manera autodidacta, segundo: ser el editor de sus propias traducciones y tercero: haber podido transformarse en difusor de pensamientos plurales en un medio ambiente caracterizado por lo contrario, es decir, por una homogeneización deseada e implementada desde el poder del pensamiento social.

La mayoría de los traductores profesionales que no encajan en ese orden establecido no tienen otra opción que emigrar o consagrarse a profesiones sin impacto en la sociedad. Navarro decidió desde muy temprano quedarse y de alguna manera repetir la odisea de Sísifo, más, a decir verdad, con mejores

\footnotetext{
${ }^{10}$ Por ejemplo, un concepto como el de la semiótica del sujeto a partir de los trabajos de Zara Mints, Dmitri Segal y Yuri Lotman, todos de la Escuela semiótica de Tartu-Moscú, lo que permitirá a Navarro escribir un libro sobre la semiótica de José Martí (inédito), del cual un capítulo ha sido publicado en España, y más recientemente en Cuba en otra antología de textos teóricos. (Navarro, 2006 [2000], pp. 139-188). Ver también: (Navarro 1986b).

${ }^{11}$ La Asociación cubana de traductores e intérpretes consigna entre sus objetivos programáticos: “2) Elevar la preparación ideológica, política, profesional y científica de sus miembros mediante la superación sistemática y permanente”. No solamente el contenido, sino también el orden de prioridad de los objetivos refleja el rol central de la ideología en la profesión bajo el actual gobierno cubano. Ver: Asociación cubana de traductores e intérpretes en las referencias.

${ }^{12}$ Quisiera traer aquí como ejemplo la declaración que hizo Fidel Castro el 10 de octubre del 2010 al afirmar en una de sus reflexiones a propósito del libro Las guerras de Obama, de Bob Woodward (Woodward, 2010): «Me comuniqué con una de nuestras mejores traductoras de inglés, rogándole un esfuerzo especial para sintetizar el contenido del mismo. El voluminoso ejemplar en ese idioma, titulado "Las guerras de Obama”, cuenta con 33 capítulos y 420 páginas. Debo señalar que en sólo tres días me entregó una síntesis de los 33 capítulos, en 99 páginas con letra de 18 puntos». (Castro, 2010). Mi comentario es el siguiente: el esfuerzo exigido en ese tipo de casos es siempre « especial ». el traductor, en el mejor de los casos para él mismo es un convencido de la doctrina oficial, es decir un instrumento útil y dócil de los políticos revolucionarios. Ese tipo de trabajo de traducción para el jefe de estado (no se puede pensar que Fidel Castro no tenga aun hoy en día similar influencia que cuando dirigía personalmente el país), debe ser hecho como norma en un tiempo récord.
} 
resultados que el legendario héroe griego. Su éxito se debe a las diferentes tácticas adoptadas en cada etapa vivida dentro del proceso sociopolítico cubano. Traducir textos teóricos de autores rusos en Cuba en los años 1970 era la única traducción posible de teoría extranjera. Navarro emprendió esa tarea porque la obra de esos autores rusos ya poseía un cierto reconocimiento no solamente en la URSS y los países aliados de ella, sino también y tímidamente, en otras latitudes. De modo que a la pregunta ¿qué autores y que textos traducir?, el traductor cubano no busca una respuesta fácil, sino que escoge aquellos que poseen una complejidad elevada, aprovechándose también del hecho de que en Cuba, en los ámbitos gubernamentales y editoriales, existe aún a principios de los años 1970 , un cierto desconocimiento ${ }^{13}$ de qué autores rusos y soviéticos son los "políticamente correctos" para el partido comunista soviético y cuales no tanto, pero eso cambia cuando llegan a la isla, hacia finales de esa década, los asesores soviéticos en el campo de la cultura y con la institucionalización de los contactos entre los departamentos ideológicos a todos los niveles (Navarro, 2009: xii).

Navarro ha publicado antologías del pensamiento ruso previas a la de 2009, en particular de textos de la Escuela de Tartu-Moscú, pero no en Cuba. En la introducción al número 2 de la revista electrónica española Entretextos, consagrada a “Lotman desde las Américas”, su director, Manuel Cáceres escribía:

Cuando ya estaba avanzada la edición de Entretextos con estos ocho trabajos, Desiderio Navarro me ofrece la posibilidad de publicar la versión electrónica del número monográfico que él seleccionó, tradujo y editó, en 1993, para la revista mexicana Escritos, del Centro de Ciencias del Lenguaje de la Benemérita Universidad Autónoma de Puebla, con el título genérico "La Escuela de Tartu. Homenaje a Iuri M. Lotman" (Cáceres, 2003).

Para entonces Navarro había tenido que publicar ya varias veces en el extranjero sus traducciones de autores rusos, porque en Cuba no podía encontrar ni el apoyo ni la autorización. La edición y publicación en Cuba de una antología del pensamiento ruso era pues un proyecto que mantuvo "engavetado" durante mucho tiempo y cuando en 2009 pudo por fin realizarlo, porque la Feria internacional del libro de La Habana estaba consagrada a Rusia, él supo adaptar el proyecto a las necesidades y circunstancias del momento, lo cual vamos a tratar de mostrar a través del análisis de algunos de esos textos.

Lo que había sido al principio tan sólo un modesto aporte de traducción teórica de autores no oficiales del Bloque del Este, con una distribución limitada a investigadores y estudiantes de las artes de

\footnotetext{
${ }^{13}$ Navarro en su prefacio a la antología es mucho más agudo en ese sentido, él lo califica de “[...] docta ignorancia inicial de las «vacas sagradas» del pavonato sobre el currículum vitae y status oficial de esos autores y a su ingenua suposición de que la política cultural en la vida académica de esos países era tan dogmática y dada a la represión administrativa como la que ellos estaban implementando aquí" (Navarro, 2009: xii).
} 
La Habana, va poco a poco creciendo en importancia y en alcance ${ }^{14}$. Los cientos de miles de ejemplares de libros teóricos del marxismo ortodoxo ruso (filosofía, sociología, y crítica de diferentes ámbitos del arte) que inundaban las universidades y librerías cubanas, provenientes de editoriales en lenguas extranjeras de la URSS o traducidos y publicados en Cuba por las editoriales estatales locales, son hoy textos obsoletos que hasta han sido reciclados de una manera muy cubana y original ${ }^{15}$. Los textos traducidos por Navarro, así como los diferentes números de la revista Criterios que él dirige son hoy, por el contrario, objetos de colección, revendidos por libreros de ocasión en pesos convertibles ${ }^{16}$ bajo la categoría de libros raros. Difíciles de encontrar en las librerías, incluso el mismo día de publicación, y a pesar de salir al mercado local con precios elevados (alrededor de 30 pesos en moneda nacional un libro o número de la revista), las ediciones se agotan rápidamente. Esos textos son citados con frecuencia por la crítica literaria cubana y en las tesis universitarias ${ }^{17}$. El contraste con los textos dogmáticos del mismo origen es revelador.

Los cinco textos que analizaremos a continuación ofrecen un panorama bastante condensado de los autores y temas que Navarro ha querido ofrecer al lector cubano en esa ocasión. Se trata de Boris Groys, Yuri Lotman, y Mijaíl Bajtín. Los temas tratados son el stalinismo en el campo estético, los procesos de brujas o la semiótica del miedo, el concepto de semiosfera, el concepto de dialogismo, y el rol del escritor y el artista en la sociedad.

\footnotetext{
${ }^{14}$ Fernando Martínez Heredia, investigador cubano del circuito oficial ha afirmado incluso que "con Criterios hay día crece, se desarrolla y multiplica un arma necesaria para Cuba y su Revolución: la polémica, para la que hay que ser "inquisitivo, crítico, audaz, honesto y no temer a equivocarse"”, (cita en: Fernández Diéguez, 2011: 12). Elogios como ese Navarro también los ha recibido antes en la isla, pero ello no ha impedido que las autoridades culturales y políticas cubanas traten de reducir o de impedir el impacto social de la actividad del traductor, de su revista y centro cultural, cuando la actividad de estos se "pasa de la raya" de la tolerancia oficial. Navarro ha denunciado en varias ocasiones esas acciones, tanto en sus propios escritos, como más recientemente en mensajes a su lista internacional de suscritos a las actualizaciones de Criterios.

15 En no pocas ocasiones al comprar un cucurucho de maní a cuentapropistas en las calles de La Habana noté que el papel utilizado había sido arrancado a manuales soviéticos de filosofía marxista-leninista o de otros libros "teóricos" de ese mismo origen, textos que se dejaron de utilizar en Cuba en el currículo educativo desde la desaparición de la URSS en 1991.

${ }^{16}$ En Cuba circulan dos tipos de moneda, el peso nacional, en el cual reciben sus salarios la mayoría de los cubanos y el peso convertible, (de un valor similar al dólar norteamericano). El salario mensual promedio de un trabajador en la isla es de 250 a 300 pesos en moneda nacional, lo cual equivale a 15 o 20 pesos en moneda convertible.

${ }^{17}$ Ver la biografía de Navarro en el sitio Cuba literaria, del ministerio de cultura de Cuba. Ver también: Arango, 2010.
} 


\section{Cinco textos de la antología cubana de Navarro}

\section{1. «El Stalinismo como fenómeno estético» de Boris Groys ${ }^{18}$.}

Artículo inaugural de la antología de Navarro, muestra desde su título el rol del traductor como agente de discusión de los términos, y ello tanto desde un punto de vista terminológico como social. Tradicionalmente en español el concepto stalinismo ha sido traducido como estalinismo ${ }^{19}$. Navarro prefiere la ortografía más cercana al ruso, o al francés y al inglés y a las principales lenguas europeas. Uno puede preguntarse si el traductor desea con ese cambio ortográfico que el lector considere la posibilidad de una visión actualizada del concepto político de marras, conociendo en particular, el contexto en el cual este concepto va a operar ${ }^{20}$. El texto de Groys diseca metódicamente el fenómeno del stalinismo en el campo de la cultura. El estilo descriptivo, imparcial, casi medical ${ }^{21}$ de Groys, permite al traductor y editor cubano avanzar en su voluntad de ir más allá de los límites ideológicos que le impone el partido comunista cubano. Navarro tiene además una razón particular para escoger este texto como la punta de lanza de la antología. Groys analiza en él una frase célebre de Stalin, relacionada con la humillante pregunta que le hacen varios escritores soviéticos al todopoderoso primer secretario del partido bolchevique, aterrorizados ante la creciente censura y represión de los años 1930: ¿cómo debemos

\footnotetext{
18 Publicado originalmente en ruso bajo el título: «Stalinizm kak esteticheskii fenomen», en Sintaksis, $1987, \mathrm{n}^{\circ} 17$. Originalmente traducido y publicado por Navarro bajo el título: “El stalinismo como fenómeno estético" Criterios, La Habana, 2008.

${ }^{19}$ Ver la definición de estalinismo del Diccionario de la Real Academia Española.

${ }^{20}$ La antología es publicada en el 2009, fecha en que no solamente Rusia es la invitada de honor a la Feria internacional del libro de La Habana, sino también la de un nuevo realineamiento geopolítico de La Habana, tras la Rusia de Putin, esta vez estando Cuba dirigida por Raúl Castro, quien nunca escondió sus preferencias ideológicas y hasta culturales por el Gran hermano ruso.

${ }^{21}$ Es representativo de esta afirmación el fragmento en que Groys dice: "El arte de la vanguardia era perseguido y aniquilado en la época staliniana en nombre de «el único método creador fructífero de la actualidad : el método del realismo socialista (o, abreviadamente, el sotsrealizm)» consistente, según la formulación oficial, en «la aspiración a mostrar la vida en su desarrollo revolucionario» [...] Una censura estética tan despiadada, hasta la aniquilación de los portadores de la estética «extraña», sin querer sugiere que el objetivo de la misma es la creación de cierto canon artístico suficientemente meditado y rigurosamente definido, (2009: 2-3).
} 
escribir? dijeron ellos. Stalin habría respondido: escriban la verdad ${ }^{22}$. Queda claro que los escritores soviéticos que vieron en el artículo determinado "la" un posesivo "mi" escondido, pues sencillamente sobrevivieron. Los que por el contrario osaron interpretar literalmente esta consigna, corajudos o ingenuos, terminaron en los gulags o el paredón. Veamos ahora la relación de estos hechos con Cuba

En 1961, a raíz de una reunión con los intelectuales cubanos en la Biblioteca Nacional de La Habana, Fidel Castro, praeceptrix imitantis, dijo: “dentro de la Revolución, todo, contra la Revolución, nada" (Castro, 1961). Desde entonces esta frase constituye el leitmotiv o versículo preferido (Navarro, 2006 [2000]: 9) de toda la política cultural cubana. Los intelectuales cubanos que interpretaron esas palabras como significativas de que al interior del discurso castrista todo estaba permitido, pero que contra el mismo todo quedaba prohibido, y habiendo sido consecuentes con tal línea creativa y política, han visto sus carreras prosperar y recibir honores y privilegios. Aquellos que se mostraron indiferentes o aun ligeramente críticos de tal postulado conocieron el ostracismo (José Lezama Lima, Virgilio Piñeira, Antón Arrufat y tantos otros), los que se opusieron, aún si de forma pacífica, pasaron por los campos de la UMAP y las prisiones (los dos casos más célebres son Reinaldo Arenas y Heberto Padilla, pero vale la pena mencionar también a Pablo Milanés, quien recientemente ha comenzado a pronunciarse al respecto $^{23}$ ). Por otro lado, un número incontable de intelectuales cubanos de todas las generaciones post 1959 se encuentran en el exilio ${ }^{24}$ por y para poder crear en libertad y disentir, si así lo consideran necesario. El texto de Groys entonces permite hacer una razonable comparación con la realidad cubana y comprender que en el contexto de la isla un paso indispensable a realizar, para poder implementar un

\footnotetext{
${ }^{22}$ Groys cita este intercambio con Stalin, algo que es bastante conocido en el contexto ruso actual y en el antiguo Bloque del Este, pues de hecho es el marxista no ortodoxo húngaro Györg Lukács quien la menciona en su texto « Le grand octobre 1917 et la littérature contemporaine », subrayando: « «Écrivez la vérité », a-t-il [Staline] un jour conseillé aux écrivains. Mais « écrivez la vérité » signifiait pratiquement écrire en accord avec les dernières résolutions du Comité central » (Lukács, 1967) (“"Escriban la verdad", dijo un día Stalin a los escritores, pero "escriban la verdad" significaba prácticamente escribir de acuerdo con las últimas resoluciones del Comité Central”). Existe una traducción catalana de este texto, ver: Lukács, 1973. Dicha "recomendación" había sido hecha por Stalin a los escritores soviéticos con motivo del primer congreso de la Unión de escritores de la URSS en 1934 (Navarro, 2009: 4). Es sintomático que algunos años más tarde, con ocasión del Pacto Molotov-Ribbentrop, firmado el 23 de agosto de 1939, esta vez ya no escritores sino oficiales de la propaganda del Ejército Rojo se hayan hecho la misma pregunta con respecto al fascismo. La respuesta aún cuando indirecta es reveladora de la esencia del stalinismo: "Queda prohibido realizar agitación y propaganda contra el fascismo ya que nuestro gobierno no ve contradicción alguna con el fascismo”. En el original: « Агитацию и пропаганду против фашизма нельзя проводить, т.к. наше правительство не видит никаких разногласий с фашизмом ", citado en (Rubzov, 2009). Rubzov es doctor en ciencias históricas, académico de la Academia de ciencias militares de Rusia, y miembro de la Asociación internacional de historiadores de la Segunda Guerra Mundial.

23 Ver la entrevista a Pablo Milanés: “La apertura cubana es un maquillaje”, en El País.

${ }^{24}$ La bibliografía en torno a las conflictivas relaciones entre el poder revolucionario y la intelectualidad cubana es bastante amplia. Nos limitamos aquí a mencionar aquella que el propio Navarro ha publicado o coordinado y que se encuentra en las referencias del presente artículo: Navarro 1989, 2002 a et 2002 b, et 2007 a.
} 
cambio social, es la eliminación del derecho al veto de uno u otro Castro sobre aquello que los ciudadanos pueden pensar, decir, escribir y publicar. Esta idea puede parecer elemental, pero a los ojos de los cubanos es vital, dado que durante más de 50 años, y sin visos de cambiar o desaparecer, ese derecho de veto ha sido ejercido y continúa siéndolo, como línea directriz de la política cultural cubana. El texto de Groys va directo al centro del debate cultural en Cuba, razón por la cual su lugar en la antología estaría más que justificado.

\section{2. «La caza de brujas: Semiótica del miedo», de Yuri M. Lotman ${ }^{25}$.}

Presentar a Lotman no es una tarea difícil. En ciertos medios académicos españoles y latinoamericanos se le considera la figura más importante de la semiótica mundial de la segunda mitad del siglo XX (Cáceres, 2011). La importancia de su obra es hoy reconocida por la comunidad científica internacional. Existe, por ejemplo, un Instituto lotmaniense de la cultura rusa en la Universidad de Bochum $^{26}$, en Alemania. Entre los numerosos autores rusos del siglo XX que Navarro ha traducido, Lotman ocupa un lugar especial, se podría decir incluso que ha sido el autor en el que más se ha interesado. La revista Criterios ha publicado trabajos de Lotman, traducidos por Navarro en los números 5-12, 21-24, 30, 31, 32 y 35, así como en la antología Textos y contextos I (1986a), editada también en Cuba. Casi todas las traducciones que Navarro ha hecho de textos lotmanienses fueron reunidas en la antología en tres volúmenes de su obra teórica La Semiosfera (Lotman, 1996, 1998 y 2000), publicada en España. Lotman, invitado por Desiderio Navarro, participó en el Primer encuentro internacional de Criterios que tuvo lugar en La Habana en 1987.

El texto original de Lotman que analizamos aquí fue publicado con posterioridad al fallecimiento del autor y el paralelismo que se puede establecer entre sus postulados fundamentales y la realidad cubana contemporánea es muy revelador. Lotman analiza un hecho histórico del Renacimiento, la recrudescencia de la "caza de brujas" que tiene lugar en paralelo con el progreso alcanzado en los campos de la ciencia y la cultura en la Europa, tanto católica como protestante. Lotman constata allí que

\footnotetext{
${ }^{25}$ Publicado originalmente en ruso bajo el título: «Ojota za ved'mami. Semiotika straja», en Semeiotiké. Trudy po znakovym sistemam, vol. 26, 1998, pp. 61-80. Originalmente traducido y publicado por Navarro bajo el título: «La caza de brujas: Semiótica del miedo» Criterios. La Habana, no. 35, 2006.

${ }^{26}$ Ver: Seminar für Slavistik/Lotman-Institut. Lotman fue vicepresidente de la Asociación Internacional de Semiótica (IASSAIS) de 1969 hasta 1984 y luego miembro de su comité ejecutivo hasta 1992. Entre muchos otros honores, en 1977 se le hizo miembro correspondiente de la Academia Británica; en 1987, miembro de la Academia de Ciencias de Noruega; en 1989, miembro de la Academia Real de Suecia y en 1990, se le entregó el título de Doctor honoris causa de la Universidad libre de Bruselas. Ver: Sección Autores de la revista Criterios en línea.
} 
“[...] la primera idea básica sobre las brujas puede ser formulada así: las brujas son una peligrosa minoría organizada" (Lotman en Navarro, 2009, Vol. 1: 24). El lector advertido podrá asociar el temor de un poder que depende de la afirmación exclusiva de una ideología, a las minorías organizadas, pero sobre todo se puede asociar el método de los procesos realizados a las brujas en el siglo XVI a aquellos de Moscú en 1937 o al caso Padilla ${ }^{27}$ en la Habana de 1972, por citar sólo dos casos. El fenómeno que subraya Lotman, citando a Jean Delumeau (Delumeau, 1978: 239-240), a propósito del síndrome de ciudad sitiada, es perfectamente reconocible en las condiciones pasadas y presentes de Cuba, donde la élite revolucionaria en el poder estimula y torna desde muy temprano en herramienta preferida ese método persuasivo, para instalar el miedo entre los intelectuales y poder así controlarlos. El gobierno cubano manipula igualmente el miedo de la población a una amenaza de invasión "permanente" de parte de los Estados Unidos, inclusive después del anuncio bilateral de restablecimiento de relaciones del pasado 17 de diciembre del 2014. Todo ello ha contribuido históricamente a desviar la atención pública de numerosos y críticos problemas internos, pero con menor eficacia a medida que pasa el tiempo. El texto de Lotman ofrece al lector sutiles herramientas de análisis de la realidad, en particular de análisis histórico comparativo, lo cual permite visualizar críticamente políticas e ideologías que no son "naturales", como se ha querido hacer creer, sino el resultado de construcciones humanas en circunstancias históricas concretas. En Cuba en particular este texto ha contribuido a los esfuerzos de estudiantes e investigadores en ciencias sociales por alejarse de la doctrina castrista y a desarrollar un pensamiento crítico propio ${ }^{28}$.

\footnotetext{
${ }^{27}$ Heberto Padilla, poeta e intelectual cubano, es considerado por algunos críticos como el "arquetipo del poeta talentoso, que predica las virtudes del individualismo en una sociedad completamente amordazada” (Lucien, 2006: 73). Padilla ganó en 1968 con su libro Fuera del juego, el premio de poesía de la Unión de escritores y artistas de Cuba (UNEAC), cuyo jurado estaba compuesto por cubanos y extranjeros, pero su libro fue publicado con un prefacio de protesta de los responsables de la UNEAC (Lançon, 2000). A continuación, una campaña difamatoria contra Padilla se orquestó en los medios de difusión del ejército cubano y en 1972 Padilla fue arrestado y obligado a hacer un mea culpa público al estilo de los procesos de Moscú de 1937. Esos hechos colmaron la copa de muchos simpatizantes en todo el mundo y provocó que numerosos intelectuales europeos y latinoamericanos, entre los cuales en las Américas se encontraban Mario Vargas Llosa, Octavio Paz, Juan Rulfo e inclusive Julio Cortázar, y en Europa, Jean Paul Sartre, Nathalie Sarraute, Susan Sontag, Marguerite Duras, hasta entonces defensores del gobierno castrista, iniciaran un largo proceso de desencantamiento y de crítica de la revolución cubana.

${ }^{28}$ En ese sentido se expresan varios autores, incluso aquellos que pertenecen al circuito oficial cubano. Dalia Acosta, periodista de IPS (una agencia de prensa cubana) refiriéndose a Navarro lo caracteriza como "a writer who became the informal coordinator of the polemical discussions among Cuban intellectuals" (Acosta, 2008). Dimitri Pietro-Samsonov, profesor universitario en La Habana, también ha dicho de las obras de Navarro que "Many writings by him have become immediate classics once published”, (Prieto-Samsonov, 2009). Se notará no obstante, que ambas opiniones han sido expresadas en inglés y publicadas en línea, de manera que eso las aleja de un acceso masivo en la propia isla, pudiéndose concluir que no están originalmente destinadas a un público cubano.
} 


\section{3. «Acerca de la semiosfera», de Yuri M. Lotman²9.}

Luego de la aparición de éste artículo en 1984, el concepto de la semiosfera adquirió reconocimiento internacional y ha constituido uno de los aportes científicos más importantes tanto de Lotman, como de la escuela de Tartu-Moscú que él fundara. El término se ha desplazado de un campo terminológico al otro, transformándose en un concepto metadisciplinario (Torop, 2006). También posee un aspecto metodológico importante que consiste en el pasaje del análisis estático al análisis dinámico, y es un texto importante en el contexto cubano, pues permite comprender por qué la monopolización de la semiosfera cubana por un solo discurso político ${ }^{30}$ ha aislado la isla del resto del continente ${ }^{31}$ y del mundo. Este concepto permite al observador extranjero analizar, con herramientas críticas esa cierta unanimidad proclamada en la vida socio-política cubana como factor legitimante del régimen. En ese texto el tratamiento por Lotman del concepto de "frontera", va en el mismo sentido al señalar que: "La función de toda frontera [incluso] la frontera de la semiosfera, se reduce a limitar la penetración de lo externo en lo interno, a filtrarlo y elaborarlo adaptativamente" (Lotman, en Navarro, 2009: 312). Este "adaptación" o "filtraje" de todo lo que proviene del exterior ha caracterizado la política ideológica, científica, cultural, y hasta pedagógica del gobierno cubano y las instituciones de educación estatales (las únicas existentes), a todos los niveles.

Otra extremadamente corrosiva conclusión lotmaniana, nos recuerda en el contexto cubano el concepto de Yurchak acerca del "principal del discurso". Aparece en el texto de Lotman de manera bastante críptica, pues no hay que olvidar las propias circunstancias del autor, pero pronto se hace clara la similitud:

Si una de las estructuras nucleares no sólo ocupa la posición dominante, sino que también se eleva al estadio de la autodescripción y, por consiguiente, segrega un sistema de metalenguajes con ayuda de los cuales se describe no sólo a sí misma, sino también al espacio periférico de la semiosfera dada, entonces encima de la irregularidad del mapa semiótico real se construye el nivel de la unidad ideal de éste (Lotman en Navarro, 2009: 315).

\footnotetext{
${ }^{29}$ Publicado originalmente en ruso bajo el título: «O semiosfere», en Semeiotiké. Trudy po znakovym sistemam, Tartu, Tartu Riikliku Ülikooli Toimetised, $\mathrm{n}^{\circ}$ 17, 1984, pp. 5-23. Originalmente traducido y publicado por Navarro bajo el título: « Acerca de la semiosfera " Criterios, edición especial. México, 1993.

${ }^{30}$ Además de la referencia hecha anteriormente al concepto de "principal del discurso" en el contexto cubano, es oportuno recordar el concepto bourdieusiano de la "monopolización del monopolio" y el paralelismo que existe entre el monopolio estatal de la violencia física y simbólica y la lucha por el monopolio de las ventajas de ese monopolio (Bourdieu, 1994: 131-135), lo cual permite profundizar el análisis contextual, alejándonos de la idea de un personalismo exclusivo como causa de los procesos sociales en Cuba.

${ }^{31}$ Diferencia que se puede observar también en relación a los aliados políticos más cercanos del gobierno cubano. La Venezuela de Chávez y de Maduro, por ejemplo, no han exigido, al menos hasta el momento, la dirección de la sociedad por un partido único, como ha sido el caso de Cuba desde bien temprano en los años 1960.
} 
La defensa a ultranza de una interpretación "nuclear" de la unidad nacional cubana ha sido el arma ideológica preferida para mantener el control del espíritu contestatario de la población ${ }^{32}$.

Un último aspecto que merece ser mencionado y es la idea de Lotman en torno a la expansión de ciertas artes y la interrupción de otras durante ciertas épocas históricas. Lotman escribe:

[...] en la historia de la cultura se pueden distinguir períodos en los que tal o cual arte, hallándose en el punto más alto de su actividad, transmite [transliruet] sus textos a otros sistemas semióticos. Sin embargo, esos períodos son relevados por otros en los que ocurre como si la rama [rod] dada del arte pasara «a la recepción» [...] basta con que nos planteemos el objetivo de describir el conjunto de las artes en los marcos de tal o cual época, para que descubramos claramente la expansión de unas y «como interrupciones» en la historia de otras. (Lotman en Navarro, 2009: 319).

En Cuba se observa desde hace ya más de un cuarto de siglo un "boom” de las artes plásticas acompañado de un mensaje que ha sido el más crítico respecto a la sociedad y al poder. Vienen a confirmar esta afirmación los sucesos alrededor del planificado performance por la artista plástica cubana de amplio reconocimiento internacional, Tania Bruguera, titulado, "El susurro de Tatlin \#6" que debió tener lugar en la Plaza de la revolución en La Habana. Este evento plástico-social fue no sólo censurado por las autoridades cubanas, sino que su autora fue en varias ocasiones arrestada y hasta el momento en que se escribe este artículo, no puede salir del país, pues arriesga no poder regresar después ${ }^{33}$.

En contraste, la literatura cubana ha sido más bien benévola ${ }^{34}$ con el discurso dominante del régimen, y queda claro que también han sido los autores literarios disidentes quienes más han sufrido las consecuencias de sus tomas de posición, teniendo que exiliarse para poder seguir creando y publicando. Este comportamiento diferenciado de estas dos artes en la Cuba contemporánea muestra la pertinencia de la tesis lotmaniana para el contexto de la isla y permite profundizar en las razones por las cuales unas u otras manifestaciones artísticas toman la vanguardia en épocas diferentes. Todos esos elementos sumados al hecho de que Navarro ha traducido casi toda la obra de Lotman, convirtiéndose en un vasto

\footnotetext{
${ }^{32}$ Fernando Ortíz en su bastante célebre El pueblo cubano, describía con minucia los rasgos característicos del pueblo de la isla y con una crítica frecuentemente corrosiva de la identidad nacional reconocía que: " "La escasa sociabilidad nuestra, la cual hace rara la unión fuerte, íntima y constante de nuestros convivientes para finalidades políticas, nos hace repudiar toda subordinación a principios superiores; por otra parte, la autoridad, al hallar el vacío a su alrededor, tiende a rectificar su incoherencia con la masa social con actos de fuerza, que distancia más y más a una de la otra. » (Ortíz, 1997: 70). Actos de violencia física e ideológica estatal sobre la población cubana han sido omnipresentes en la historia de Cuba. En ciertos periodos han prevalecido los primeros y en otros los segundos.

${ }^{33}$ Ver el perfil de Tania Bruguera y la notica de su arresto.

${ }^{34}$ Es preciso recordar que autores de reconocimiento internacional (publicados o no en Cuba), y que viven en la isla, como son: Leonardo Padura, Pedro Juan Gutiérrez y otros, no cuestionan directamente el orden sociopolítico imperante allí, aun cuando puedan condenar algunas de las aberraciones individuales de ese mismo orden.
} 
conocedor y experto de la misma, explican exhaustivamente la presencia de estos dos textos en la antología.

\section{4. «El autor y el héroe en la actividad estética », de Mijaíl M. Bajtín. ${ }^{35}$}

Texto seminal de Bajtín, fue escrito en la primera mitad de los años 1920, pero publicado mucho más tarde, en una selección de artículos póstumos titulada La Estética de la creación verbal (1979). Texto inconcluso, es no obstante representativo de los rasgos filosóficos de su época, en particular del neokantismo de Herman Cohen, de la Escuela de Marbourg. Se presenta en él lo que será el aporte fundamental de este autor a la teoría literaria, es decir, el concepto de dialogismo, aplicado tanto a la literatura, como a la lengua o al pensamiento humano en general.

En nota del traductor a pie de página Navarro señala que este texto había sido ya traducido directamente del ruso al español por Tatiana Bubnova (Navarro 2009: 284). El traductor consideró, no obstante, necesaria la retraducción, porque la publicación en traducción española de Bubnova había sido realizada a partir de la edición rusa de 1979 que no incluía, según el prologuista de la segunda edición rusa de 1986, S. G. Bocharóv: “[...] un fragmento que se había conservado del primer capítulo, el cual encierra unas tesis preliminares generales sobre el hombre como condición de la visión estética en la vida real y en el arte" (Bocharóv, en Navarro, 2009: 284). Es entonces de esta segunda edición que parte Navarro, traduciendo también el mencionado prólogo.

Varios otros factores tienen una incidencia en la selección de este texto por parte del traductor y editor de la antología. El más pertinente para el contexto de destino sería la proximidad entre dos conceptos: el dialogismo de Bajtín y la transculturación de Ortíz, así como la confrontación dialógica de ambos con sus antípodas: la homofonía y la aculturación. La transculturación hace referencia a los elementos culturales que una comunidad minoritaria determinada se apropia de la dominante, remodelándolos y adaptándolos a sus necesidades (Ortíz, 1991 [1940]). En cuanto al dialogismo, asociado igualmente a la polifonía, Bajtín lo define como « voix [qui] restent indépendantes et [qui] se

\footnotetext{
${ }^{35}$ Publicado originalmente en ruso bajo el título: «K filosofii postupka», en Filosofia $i$ sotsiologuïa nauki $i$ tejniki. Ezhegodnik 1984-1985 (1986). Moscú, Nauka, pp. 138-160. Originalmente traducido y publicado por Navarro bajo el título: «El autor y el héroe en la actividad estética», Criterios, $n^{\circ}$.31. La Habana, 1994. La biografía de Mijaíl Bajtín es bastante conocido, recordemos tan sólo que vivió en Rusia entre 1898 y 1975 y que fue uno de los teóricos e historiadores de la literatura más relevantes de su época, precursor de la sociolingüística.
} 
combinent en tant que telles dans une unité d'un ordre plus élevé que celui de $\mathrm{l}^{\prime}$ homophonie » ${ }^{36}$ (Bakhtine, 2001: 29).

La transculturación no puede ser comprendida si no es en su contexto espacio-temporal colectivo, pues está presente en la interacción de individuos de diferentes herencias culturales y en la evolución que implica la incorporación y la fusión de elementos diversos, a veces hasta contradictorios, pero que reducen esa carga negativa mediante acciones de carácter metonímico, buscando contigüidades en lugar de oposiciones. El dialogismo implica la interacción que se crea entre el discurso del narrador y los discursos de otros personajes o entre dos discursos internos de un personaje, lo cual permite crear una especie de voz y conciencia alternativas a la del narrador, pudiendo éste último mantener una posición "neutra", de modo que vemos como hay también un efecto de búsqueda de la contigüidad que se puede calificar de metonímica. La transculturación y el dialogismo se nos presentan entonces en este marco comparativo, como fenómenos culturales que comparten ciertos rasgos similares (búsquedas metonímicas), y que se distinguen por el hecho de que una es más bien de carácter colectivo y el otro de carácter individual. Ambos constituyen argumentos que propugnan una interpretación recursiva en el sentido que le da la teoría de la complejidad, es decir, que son "productores de aquello que les produce" (Morin, 1990: 100). Se contribuye de este modo a una comprensión compleja y dialógica de la realidad, que resulta crítica per se tanto de una homofonía ideológica como de una aculturación asimiladora. Ni una ni otra de estas antípodas son propias a la identidad nacional de los cubanos, parece decirnos Navarro al escoger este texto.

\section{5. «Tareas inmediatas de los estudios literarios », de Pavel N. Medvedev et Mijaíl M. Bajtín. ${ }^{37}$}

Este último texto que analizaremos aquí constituye el segundo capítulo del libro de Medvédev/ Bajtín, El Método formal en la literatura. La decisión de incluir en la antología este texto fundador de Bajtín va en la dirección de una preocupación recurrente durante toda la carrera del traductor y editor cubano: reconsiderar el rol del escritor y el artista en la sociedad, por ser éste pionero en la detección in statu nascendi de los problemas ideológicos que se encuentran en proceso de formación en una sociedad dada. Navarro trata de revindicar de esta manera el rol del intelectual crítico en la suya propia. El

\footnotetext{
36 "Voces [que] se mantienen independientes y [que] se combinan como tales en una unidad de un orden más elevado que el de la homofonía"

${ }^{37}$ Publicado originalmente en ruso bajo el título: «Ocherednie zadachi literaturovedeniia», en Pavel N. Medvédev, Formal’nyi metodv literaturovedenü. Leningrad, 1928. (Navarro realiza su traducción a partir de la reimpresión realizada por la editorial Hildesheim, New York, 1974: 27-55). Originalmente traducido y publicado bajo el título: «Tareas inmediatas de los estudios literarios», (Navarro, 2009, Vol.2: 218).
} 
contexto político en el que él opera habiéndose caracterizado por la restricción sistemática de la libre participación y muy en particular, de la participación crítica del intelectual en los asuntos públicos y de orden político. Bajtín, una vez más, le sirve de punta de lanza.

En su texto, el autor ruso insiste en la independencia y las particularidades ideológicas de la literatura y el arte en la sociedad y condena el reduccionismo al que esas manifestaciones pueden ser sometidas por el poder político. Para Bajtín, la literatura tiene un estatus de ideología en proceso de formación y él señala "tres errores metodológicos fundamentales" de los críticos e historiadores de la literatura: 1) limitar el arte y la literatura al simple papel de "sirvientas" y de transmisoras ideológicas; 2) reducir la obra literaria y artística a un panfleto político y a declaraciones de principios, sin tener en cuenta que el contenido de la obra literaria refleja tan sólo un "horizonte ideológico"; 3) dogmatizar y absolutizar los elementos ideológicos básicos reflejados por el artista en el contenido de su obra (Bajtín en Navarro, 2009, Vol. 2: 221), pero Bajtín va más allá y desarrolla otra idea fundamental:

[...] en el horizonte ideológico de cualquier época y cualquier grupo social no hay una sola verdad, sino varias verdades que se contradicen mutuamente, y no hay un solo camino ideológico, sino varios caminos ideológicos divergentes (Bajtín en Navarro, 2009, Vol.2: 222).

En un país donde el pluralismo ha sido vetado por ley (ya que no puede haber en Cuba según la Constitución de $1976^{38}$ otro partido e ideología que la comunista) ${ }^{39}$ se comprende el peso de tales enunciados. Se ha podido constatar igualmente cómo esos propósitos de Bajtín han influenciado el pensamiento de Navarro. Por ejemplo, el ciclo de conferencias organizada por el Centro cultural Criterios que él dirige, fue el punto culminante de su reflexión en torno a "La política cultural del periodo revolucionario: memoria y reflexión”, título también de la publicación colectiva ulterior (Navarro, 2007b). Su presentación en la misma, titulada “¿Cuántos años de qué color? Para una introducción al Ciclo" (Navarro, 2007b: 15-24), constituye una contribución teórica importante para el debate intelectual cubano de ese año y hasta hoy. La razón principal es que este texto pone sobre el tapete del debate público los cuatro modelos de sociedad y de cultura en lucha en la Cuba contemporánea. Ello lo transforma en el cuestionamiento teórico y político más relevante propuesto hasta el día de hoy en la isla, ya que constituye un ataque frontal al dilema político más agudo y general de todos los cubanos: ¿qué modelo de sociedad estamos construyendo? Navarro explica:

\footnotetext{
38 Ver: Constitución de la República de Cuba.

${ }^{39}$ El artículo 5 de la constitución de 1976 con las enmiendas de 1992 estipulan: “artículo 5o.- El Partido Comunista de Cuba, martiano y marxista-leninista, vanguardia organizada de la nación cubana, es la fuerza dirigente superior de la sociedad y del Estado, que organiza y orienta los esfuerzos comunes hacia los altos fines de la construcción del socialismo y el avance hacia la sociedad comunista".
} 
1. lo que Marx llamó «comunismo de cuartel», (monismo artístico: exigencia de un arte apologético y acrítico, el artista sólo como entretenedor, ornamentador o ilustrador de tesis);

2. socialismo democrático (diálogo artístico, con inclusión y fomento de un arte crítico-social);

3. capitalismo de Estado o «socialismo de mercado» (pluralismo artístico, con exclusión de un arte críticosocial, apertura a la globalización americanocéntrica y fomento de la cultura destinada al mercado transnacional y nacional);

4. capitalismo neoliberal (sumisión del arte al mercado transnacional y nacional; neutralización y recuperación de un eventual arte crítico-social por el mercado) (Navarro, 2007b: 20).

La tipologización de los cuatro modelos de sociedad en lucha en Cuba, -recordemos las ideas de Bajtín ya citadas sobre las "varias verdades que se contradicen mutuamente" generando "varios caminos ideológicos divergentes" y con mayor relevancia aun la idea navarriana sobre el "diálogo artístico" en el modelo del "socialismo democrático", que es evidentemente el que él está apoyando, constituyen un buen ejemplo de la simbiosis operada en el pensamiento de este singular traductor cubano, que ejecuta una apropiación teórica del pensamiento ruso antiortodoxo y de Bajtín en particular, para marcar su huella en un debate ciudadano y cultural clave para la nación cubana.

\section{Conclusion}

El pensamiento cultural y teórico ruso-soviético tuvo una introducción forzada e impuesta en la Cuba de las décadas de 1960 y 1970. Ello fue posible gracias al capital simbólico que disfrutaba el gobierno revolucionario en esa época. Para poder hacer aceptar ese radical reajuste geopolítico, el gobierno cubano tuvo que apoyarse en el despertar nacionalista y antimperialista de su pueblo, antimperialismo que, desde el poder, fue tornándose paulatinamente en mero antinorteamericanismo. En su mayoría se trató de textos traducidos en la ex-URSS y en menor medida en Cuba, promoviendo casi siempre la ideología de un comunismo ortodoxo, de corte stalinista o neostalinista soviético o local ${ }^{40}$. El sustrato ideológico de esos textos, para finales del siglo XX estaba ya en plena bancarrota. No obstante y paralelamente a ese proceso oficialista, tuvo lugar otro proceso de traducción de textos antiortodoxos del mismo origen, que fueron creando las condiciones psicosociales e ideológicas para un cambio de mentalidad y para el análisis, por parte de las jóvenes generaciones de intelectuales y artistas cubanos, de las problemáticas que el turbulento final del siglo XX y comienzo del nuevo milenio les iba planteando de manera creciente. Desiderio Navarro fue y sigue siendo un factor decisivo en ese ciclópeo

\footnotetext{
${ }^{40}$ El stalinismo "clásico" entra en Cuba y se adapta a las circunstancias locales con el partido prerrevolucionario llamado: Partido Socialista Popular (PSP). Este va a ser uno de los tres partidos o movimientos populares que integran el poder revolucionario desde 1959. El neostalinismo cubano es construido entonces a partir de los años 1960 de la fusión de este antiguo stalinismo local, sumado a las ideas de Fidel Castro, quien detenta desde muy temprano el rol de "principal del discurso" en el contexto de la Cuba contemporánea.
} 
esfuerzo. Gracias a su trabajo sin pausa, el pensamiento teórico y cultural ruso tiene ya un lugar justamente ganado en la historia del arte y del pensamiento cultural cubano, tanto como fuente de inspiración teórica, así como también temática ${ }^{41}$. Esas traducciones han favorecido la evolución del análisis de temas de candente actualidad en la isla y se puede afirmar que en el ambiente de asfixia intelectual de la Cuba de los años 1970 los textos "rusos" de Navarro hicieron entrar un poco de aire fresco teórico. En los años 1980 el boom de las artes plásticas cubanas constituyó un producto auténtico y de reconocimiento mundial gracias en buena medida a esas lecturas. En los años 1990 con la caída de la URSS y el Bloque del Este comenzó el proceso de reivindicación de la labor navarriana, sin por ello alcanzar nunca una aceptación ni un apoyo oficial incondicional. En el nuevo siglo y milenio Navarro lucha aun contra los mismos obstáculos en una sociedad mucho más favorable a su mensaje, pero también mucho más fatigada por el largo periodo de privaciones y estancamiento. Navarro ha conseguido en esas circunstancias no sólo resistir al stalinismo y neostalinismo de factura soviética, así como al neostalinismo local, sino también desestabilizarlos progresivamente y contribuir con su persistente trabajo traductivo al deterioro del capital simbólico de esa ortodoxia. Navarro ha hecho también algo más. Con su activismo y con su trabajo teórico, ha contribuido a entrever otro proyecto de país y a levantar su discusión entre las élites culturales de la isla. Ese proyecto, el de un socialismo democrático, debería apoyarse en la recuperación del rol del intelectual crítico en la sociedad, pero Navarro es un hombre de su época, marcado a fuego por los acontecimientos de la Cuba revolucionaria de los años 1960 y 1970 y definitivamente comprometido con los postulados de un socialismo en Cuba, aun cuando el que él promulga no haya sido nunca materializado.

Los textos que él ha traducido sí han gozado, por el contrario, de una excelente recepción dentro y fuera de Cuba sobrepasando sus propias expectativas. Nuevas generaciones de intelectuales críticos cubanos en efecto han surgido, pero ellas no son ideológicamente homogéneas. No todos comparten el utópico deseo navarriano de ver a Cuba seguir el camino de un incierto socialismo, que nunca pudo ser verdaderamente construido y difícilmente lo será en un mundo de cambios geopolíticos e ideológicos cada vez más diversos, complejos y excluyentes.

La obra de Navarro es a pesar de todo de gran relevancia e impacto. En particular en América latina y en España, sus primeras traducciones al español de Bajtín, de Lotman y de tantos otros autores rusos sirvieron a difundir un pensamiento teórico que difícilmente podía haberlo sido en ese momento, debido al carácter de aislamiento que tuvo el mundo bipolar del lado del Bloque del Este. Los contactos,

\footnotetext{
${ }^{41}$ Ello se ha visto demostrado de manera fehaciente con la serie de exposiciones de pinturas y fotografías de artistas plásticos cubanos en la propia isla, que han tenido lugar desde 2011 y se han titulado: "Da Kantzá (hasta el fin); "La bota rusa" y "Carne Rusa". Ver: Soviet Cuba: Rusofilia en Cuba.
} 
las visitas, y las conexiones en el interior de la ex-URSS y los países socialistas hicieron de Navarro un interlocutor de excepción del mundo hispanoparlante ante el rusoparlante y ese rol se extendió inclusive al ámbito lusófono, en particular en Brasil, donde sus traducciones fueron operatorias para el conocimiento del pensamiento ruso.

El traductor cubano pudo entonces proveer a sus conciudadanos con visiones críticas de la realidad en un espacio de censura y de sospecha hacia toda producción intelectual occidental. Contribuyó así a alejar a su lectorado de las identidades mortíferas (Maalouf, 2001 [1998]), promovidas por el dominante discurso stalinista o neostalinista cubano. Supo mostrar que el traductor en la esfera nacional o local puede intervenir como mediador en la correlación de fuerzas entre el intelectual crítico y el poder, haciendo conocer gracias a su trabajo, opiniones, ideas y experiencias de otras culturas que son útiles para contextos como el cubano. Logró pues contribuir a crear valores cívicos, a difundir conocimientos científicos y a hacer conocer logros culturales que refuerzan la pertinencia de un acercamiento epistemológico complejo y evolutivo de la realidad (Morin, 2008) y de adaptacióninteracción no conflictiva con un mundo que "simultáneamente se globaliza, se particulariza o se transculturaliza" (Welsch, 1999: 299). 


\section{Bibliografía citada}

Asociación Cubana de Traductores e Intérpretes.

Acosta, Dalia. “The Debate Continues". IPS (2008).

Arango, Arturo. “Cuba, los intelectuales ante un futuro que ya es presente”. Temas 64 (2010): 80-90.

Bakhtine, Mikhaïl y Bahtin, Mihail Mihajlovič (2001). Problèmes de la poétique de Dostoüevski. Lausanne : Slavica - Editions l'Age d'Homme.

Bastin, Georges L.; Echeverri, Álvaro y Campo, A. "La traducción en América Latina: propia y apropiada”. Estudios. Revista de Investigaciones Literarias y Culturales 24 (2004). Caracas: Universidad Simón Bolívar: 69-94.

Bastin, Georges L. "La pertinencia de los estudios históricos sobre traducción en Hispanoamérica". Estudios Interdisciplinarios de América Latina y el Caribe 21, 1 (2010).

Blanc, Hélène y Lesnik, Renata (2009). Les prédateurs du Kremlin (1917-2009). Paris : Seuil.

Bourdieu, Pierre (1989). La Noblesse d'État, grandes écoles et esprit de corps. Paris : Les éditions de minuit.

Bourdieu, Pierre (1994). Raisons pratiques : Sur la théorie de l'action. Paris : Seuil.

Cáceres, Manuel et alii. “Lotman desde América”. ENTRETEXTOS. Revista electrónica semestral de estudios semióticos de la cultura 2 (2003).

Castro, Fidel. "Discurso pronunciado por el comandante Fidel Castro Ruz, Primer ministro del gobierno revolucionario y secretario del PURSC, como conclusión de las reuniones con los intelectuales cubanos, efectuadas en la Biblioteca nacional el 16, 23 y 30 de junio 1961". Discursos e intervenciones del Comandante en Jefe Fidel Castro Ruz (1961).

Castro, Fidel. "El imperio por dentro, primera parte”. Reflexiones de Fidel (2010).

Charaudeau, Patrick y Maingueneau, Dominique (dirs.) (2002). Dictionnaire d'analyse du discours. Paris : Seuil.

Colón Rodríguez, Raúl Ernesto (2011). La traduction de la pensée culturelle russe par Desiderio Navarro à Cuba, 1960-2009 (tesis de maestría). Ottawa : Université d’Ottawa. 
Constitución de la República de Cuba.

Criterios (Revista y colección editorial internacionales de pensamiento teórico sobre literatura, artes, cultura y sociedad).

Cuba Literaria, revista del Ministerio de cultura de Cuba.

Delumeau, Jean (1978). La Peur en Occident, XIVe-XVIIIe siècles. Une cité assiégée. Paris : Ed. Fayard.

Fernández Diéguez, Eliécer. "La cultura cubana en la década del 70. El quinquenio gris. Un estigma para la creación artística y literaria”. Archivo Cubano (2011).

Groys, Boris. Web Personal.

Judt, Tony y Snyder, Timothy (2012). Thinking the twentieth century. New York: Penguin Press.

Lotman, Yuri (1996, 1998 y 2000). La Semiosfera (3 vols.). Madrid: Cátedra.

Lucien, Renée Clémentine (2006). Résistance et cubanité. Trois écrivains nés avec la Révolution cubaine. Paris: L'Harmattan.

Lukács, Györg. "Le grand Octobre 1917 et la littérature contemporaine”. L'Homme et la société 5 (1967): 3-15.

Lukács, Györg (1973). El gran octubre de 1917 i la literatura contemporània, València: Tres i Quatre

Maalouf, Amin (2001 [1998]). Les identités meurtrières. Paris : Le Livre de Poche.

Markiewicz, Henryk (2010). Los estudios literarios: conceptos, problemas, dilemas. La Habana: Centro teórico-cultural Criterios.

Meschonnic, Henri (1999). Poétique du traduire. Paris : Éditions Verdier.

Morin, Edgar [2005 (1990)]. Introduction à la pensée complexe. Paris: Éditions du Seuil.

Morin, Edgar (2008). La Méthode. Paris : Éditions du Seuil.

Navarro, Desiderio (ed.) (1986a). Textos y contextos. Una ojeada en la teoría literaria mundial vol. I. La Habana: Arte y Literatura.

Navarro, Desiderio (1986b). Cultura y Marxismo, problemas y polémicas. La Habana: Editorial Letras Cubanas. 
Navarro, Desiderio (1989). Ejercicios del criterio. La Habana: Ediciones UNION.

Navarro, Desiderio. “Criterios in medias res publicas”. Criterios (2002a).

Navarro, Desiderio. “30 años de Criterios: hacia una globalidad sin Centro”. Criterios (2002b).

Navarro, Desiderio [2006 (2000)]. "In Medias res Publicas: Sobre los intelectuales y la crítica social en la esfera pública cubana”. Navarro, Desiderio. Las Causas de las Cosas. La Habana: Editorial Letras Cubanas: 7-31.

Navarro, Desiderio (2007a). A pe(n)sar de todo. Para leer en contexto. La Habana: Editorial Letras Cubanas.

Navarro, Desiderio (2007b). “¿Cuántos años de qué color? Para una introducción al Ciclo”. Navarro, Desiderio y Heras León, Eduardo (eds.) La política cultural del período revolucionario: memoria y reflexión. La Habana: Centro Teórico-Cultural Criterios: 15-24.

Navarro, Desiderio (2009). El Pensamiento Cultural Ruso en criterios: 1972-2008 vol. 2. La Habana: Centro Teórico Cultural Criterios.

Ortiz, Fernando [1991 (1940)]. Contrapunteo cubano del tabaco y el azúcar. La Habana: Editorial de Ciencias Sociales.

Ortiz, Fernando (1997). Elpueblo cubano. La Habana: Editorial de Ciencias Sociales.

Padilla, Heberto (1969). Hors-jeu. Paris : Seuil.

Philippe, Lançon. “Heberto Padilla, hors-jeu. Le poète cubain est mort en exil”. Libération (2000).

Prieto-Samsonov, Dimitri (2009). “Award for a Unique Cuban”. Havana Times.

Rubzov, Iuri. “Сталин и Гитлер: от ненависти к любви и обратно” Svpressa (2009) [Rubzov, Iuri (2009). "Stalin y Hitler: del odio al amor y viceversa" Svpressa (2009].

Seminar für Slavistik/Lotman-Institut

Schleiermacher, Friedrich [1999 (1813)]. Des différentes méthodes de traduire (edición bilingüe). Paris: Seuil : 31-93.

Torop, Peeter. "The Semiosphere and/as the Research Object of Semiotics of Culture" Sign Systems Studies 33.1 (2005). 
Van Gorp, Hendrik et alii. (2001). Dictionnaire des termes littéraires. Paris: Honoré Champion.

Welsch, Wolfgang (1999). "Transculturality - the Puzzling Form of Cultures Today" Featherstone, Mike y Lash, Scott. Spaces of culture: city, nation, world. London, Thousand Oaks, California: Sage.

Yurchak, Alexei. "Soviet Hegemony of Form: Everything was forever until it was no more". Society for Comparative Study of Society and History vol. 45, n. 3 (2003): 480-510.

Yurchak, Alexei. “Поздний социализм и последнее советское поколение” [“El socialismo tardío y la última generación soviética”]. Неприкосновенный запас 2, 52 (2007). 